\title{
Psychiatric Comorbidity Among Adolescents with Substance Use Disorder
}

\author{
Niaz Mohammad Khan ${ }^{1, ~ *, ~ M S I ~ M u l l i c k ², ~ S h a r m i n ~ H u s s a i n ~}{ }^{3}$ \\ ${ }^{1}$ Department of Psychiatry, National Institute of Mental Health, Dhaka, Bangladesh \\ ${ }^{2}$ Department of Child and Adolescent Psychiatry, Bangabandhu Sheikh Mujib Medical University, Dhaka, Bangladesh \\ ${ }^{3}$ Department of Pediatrics, National Institute of Neurosciences (NINS), Shere Bangla Nagar, Dhaka, Bangladesh
}

Email address:

nmadil27@gmail.com (N. M. Khan)

${ }^{*}$ Corresponding author

\section{To cite this article:}

Niaz Mohammad Khan, MSI Mullick, Sharmin Hussain. Psychiatric Comorbidity Among Adolescents with Substance Use Disorder. American Journal of Psychiatry and Neuroscience. Vol. 9, No. 3, 2021, pp. 98-106. doi: 10.11648/j.ajpn.20210903.14

Received: July 2, 2021; Accepted: July 14, 2021; Published: August 7, 2021

\begin{abstract}
Substance Use Disorder (SUD) in adolescents, is a condition in which the use of one or more substances leads to clinically significant impairment or distress. It is a significant public health problem globally with a higher burden in low and middle-income countries. To find out the frequency and pattern of psychiatric comorbidity among the adolescents with Substance Use Disorder. This descriptive and observational study was conducted in the Department of Psychiatry, Bangabandhu Sheikh Mujib Medical University (BSMMU), Dhaka, Bangladesh from March 2016 to September 2018. The present study aimed at assessing the presence of comorbid psychiatric disorders among adolescents with Substance Use Disorder (SUD) included a total of 70 adolescents. Over half $(60 \%)$ of the respondents were $<15$ years old, and $40 \%$ of the respondents were $>15$ years age. The mean age of the respondents was 13.2 \pm 2.1 years, and the range was $11-17$ years. About $50 \%$ of the adolescents interviewed had Tobacco Use Disorder (51.4\%), followed by Cannabis Use Disorder (47.1\%). Among the respondents, 22.8\% had no psychiatric illness. The overall psychiatric disorders among adolescents were $77.1 \%$ (54 out of 70). Most of the patients (45.7\%) had more than one psychiatric disorder diagnosed. Regarding the specific disorder diagnosed Conduct Disorder was $31.4 \%$ and different socio-demographic features. Based on the findings of the study, it can be concluded that adolescents with SUD have a high rate of other comorbid psychiatric disorders.
\end{abstract}

Keywords: Comorbidity, Adolescence, Substance Use Disorder, Psychiatric Disorder, Substance Use Disorder

\section{Introduction}

A drug is a natural or synthetic chemical substance that affects living processes. Substance-related disorders are psychiatric disturbances developing during or following substance use, and attributable to it. The drugs that most often produce substance-related disorders include tobacco, alcohol, cannabis, stimulants (cocaine, amphetamine, methamphetamine, mephedrone, and others), opioids, sedative-hypnotic and anxiolytic agents, inhalants (volatile hydrocarbons), phencyclidine or other arylcyclohexylamines, and other hallucinogens. Numerous other substances, including nitrous oxide, amyl- or butylnitrite, or anabolic steroids also may produce these disorders [1]. Early initiation of substance use is usually associated with a poor prognosis and a lifelong pattern of deceit and irresponsible behavior [2]. The hope that simple information given through educational programs will be sufficient to prevent drug dependence is frequently expressed, however, there is no evidence to support it and there are many reasons to doubt it [3]. There are substantial geographic variations in drug use prevalence among the adolescents, with higher rates in higher-income countries (although data from lower-income countries often are lacking). A survey done in the United States investigated the prevalence of SUD among 203 adolescent psychiatric inpatients and found $41 \%$ met the criteria for a SUD [4]. A study was done in West Bengal, India, out of 416 students, 
$52(12.5 \%)$ used or abused any one of the substances irrespective of time and frequency in the lifetime; 26 $(15.1 \%)$ were among the urban students and $26(10.7 \%)$ were among their rural counterparts. More than two-thirds (73.07\%) of the respondents expressed a desire to quit substance use and $57.69 \%$ had tried to stop. 'Easy availability' and 'relief from tension' were the most frequent reasons for the continuation of substance use [5]. Comorbid mental disorder among adolescents with substance abuse include depression, anxiety, conduct disorder, and attention-deficit/hyperactivity disorder (ADHD) [6]. A study conducted by Shantna. K. et al., [7] show that the most prevalent comorbid disorders in substance dependence patients and substance abusers were depressive disorders. They also conclude that the majority of substance dependence patients suffered from comorbid mental disorders. Approximately $11-48 \%$ of adolescents with SUDs in community samples have co-occurring internalizing disorders, with depression co-occurring more commonly than anxiety disorders. Rates of internalizing cooccurrence are even higher in clinical samples [8]. A recent study of health records, for example, found that $29 \%$ of male and $49 \%$ of female adolescent patients with SUDs had co-occurring mood disorders, whereas $9 \%$ and $19 \%$ of male and female patients, respectively, had co-occurring anxiety disorders [9]. The community-based prevalence study of psychiatric disorder among children of Bangladesh has not yet been studied. According to the analytical predictions, prevalence would be roughly $10-20 \%$ among children and adolescents as like as the prevalence findings of the reports of the developing countries. A study of psychiatric outpatient attendance at the Institute of Mental Health and Research in Dhaka, revealed that $8.6 \%$ of cases were children or adolescents [10]. In a different analysis of psychiatric morbidity among the Institute outpatients, emotional disorder was found to be the largest group with $32.5 \%$, followed by conduct disorder $18.8 \%$; mental retardation comprised $16.2 \%$, psychoses and allied conditions $11.2 \%$, epilepsy with behavioral problems $12.5 \%$ and the rest $8.5 \%$ comprised of other groups of disorders, according to ICD-9 criteria [11]. In a survey among the children in a child guidance clinic at the Bangladesh Institute of Child Health and Dhaka Childrens' Hospital, Rabbani and Quamaruzzaman et al., [12] assessed psychiatric morbidity by using the Rutter Multiaxial diagnostic system. Their findings revealed that conduct disorder was $8.9 \%$, somatoform disorders $7.1 \%$, attention deficit hyperactivity disorder $68 \%$, autism $6 \%$, and other emotional disorder $15.28 \%$ of the cases. Specific delays in development were found to be $10 \%$, and mental retardation was $17.8 \%$ of the cases. Substance-induced depression occurs during periods of substance use but exceeding the expected effects of intoxication or withdrawal from the substance used. Primary or independent major depression is defined as either predicting substance use entirely or occurring during periods of sustained abstinence [13]. Another investigated adolescent admissions to residential substance abuse programme and reported the prevalence of psychiatric disorders comorbidity in $64 \%$ of the 91 adolescents; depression (24\%), conduct disorder (CD) $(24 \%)$ and attention deficit hyperactivity disorder (ADHD-11\%) were the most common conditions [14]. In one study, alcoholics with independent major depression were found to be more likely to attempt suicide than those with substance-induced depression [13]. Further, literature review estimate rates of psychiatric comorbidity among adolescents receiving treatments for substance abuse at 50-90\% [15]. Adolescents with SUD have higher rates of both mood disorders and $\mathrm{CD}$ [15]. ADHD, CD and substance use often co-exist and are associated with poorer treatment outcomes [14]. Those with CD are more likely to have an earlier onset of substance use [16]. Timely intervention of co-occurring psychiatric and SUDs is associated with better engagement with treatment [17]. The pattern of CODs in adolescents by race/ethnicity [18]. Similarly, there appear to be gender variations [17]. An Irish study investigated 88 adolescents attending substance abuse treatment and found that psychological problems are more common in girls compared to the boys; the girls had higher levels of low self-concept, anxiety problems, depression, anger problems and disruptive behaviors [19]. In a systematic review made in Bangladesh reports the prevalence of mental disorders in children. Earliest report among urban primary school children revealed $13.4 \%$ had some behavioral disorder, with boys being twice more affected than girls (20.4 vs. $9.9 \%$ ). However, a study among socially disadvantaged (urban slum) children, reported $22.9 \%$ had some form of psychiatric disorder with a slightly lower prevalence in boys than girls $(20.0 \%$ in boys and $25.5 \%$ in girls $)$. Another study found $14.6 \%$ of children with behavioral problems as reported by the parents in rural Bangladesh [11]. Another more recent community-based study reported the prevalence of mental disorder among $18.4 \%$ of the children [20]. The history of diagnoses in the Diagnostic and Statistical Manual (DSM) reflects the evolving concept of addiction. The term "alcoholism" was eliminated. Substance Use Disorders (SUD) got their own section and were no longer listed under personality disorders. The idea of a public health model was introduced to explain addiction. The dependence category required either tolerance or withdrawal (or both) to be present. Abuse was the presence of drug-related problems in the absence of physiological symptoms. DSM-IV (1994) includes over 100 different Substance-Related Disorders (SRD) for twelve different classes of drugs. Therefore there is a different but high magnitude of comorbid psychiatric disorder in adolescents with SUD. The study was intended to find out the different type of SUD our adolescents are suffering from in two different treatment centers and their magnitude and pattern of comorbid SUD. The expected outcome of the study is to help in the field of the adolescent SUD and its comorbidity's proper assessment, diagnosis, and formulation of an effective management plan. 


\section{Objectives}

\subsection{General}

To find out the frequency and pattern of psychiatric comorbidity among the adolescents with Substance Use Disorder.

\subsection{Specific}

I. To determine the types of substance use disorder among adolescents.

II. To evaluate the specific type and frequency of comorbid psychiatric disorder among adolescents with substance use disorder like conduct disorder, attention deficit hyperactivity disorder, oppositional defiant disorder, major depressive disorder, anxiety disorders, etc.

III. To explore the relationships between sociodemographic variables and psychiatric comorbidity among adolescents with substance use disorder.

\section{Materials \& Methods}

\subsection{Type of Study}

This was a descriptive type of cross-sectional study.

\subsection{Place of Study}

This study was conducted in the Department of Psychiatry, Bangabandhu Sheikh Mujib Medical University (BSMMU), Dhaka, Bangladesh.

\subsection{Duration of Study}

From March 2016 to September 2018.

\subsection{Sample Size}

75 (seventy five).

\subsection{Place of Data Collection}

Central Drug Addiction Treatment Centre (CDC), Tejgaon, Dhaka and Ashokti Punorbashon Nibash (APON), Singair, Manikganj, Bangladesh.

\subsection{Study Population}

Adolescents with Substance Use Disorder aged between 11 to 17 years. Only male patients were taken as the sample because the above-mentioned treatment facilities do not provide service for the female adolescents.

\subsection{Selection Criteria}

\subsubsection{Inclusion Criteria}

Children aged between 11 to 17 years who fulfill the diagnostic criteria of different Substance Use Disorder, namely alcohol, cannabis, hallucinogens, inhalants, opioids, sedatives- hypnotics- or anxiolytics, stimulants, and tobacco.

\subsubsection{Exclusion Criteria}

I. Adolescents in intoxication or withdrawal state.

II. Patients having acute medical or another condition communication was not possible.

Permission was taken from the authority of the two institutions, namely Central Drug Addiction Treatment Hospital and Ashokti Punorbashon Nibash (APON). The study subjects were given ideas about the purpose, method, and outcome of the study in brief. Data was collected by face to face interview. After getting the informed written consent from the patient and one of their parents, the diagnostic criteria for SUD, according to Diagnostic and Statistical Manual of Mental Disorder, 5th edition (DSM-5), was applied to confirm the diagnosis of SUD and type of SUD the patient suffering from. Then a semi-structured questionnaire was applied to the patient to collect the sociodemographic characteristics, family history of psychiatric disorders or diagnoses, the main substance of abuse, forensic history and educational history. The Development and Wellbeing Assessment Scale (DAWBA) self-report and parent report interview, Bangla version were applied among SUD patients to diagnose the comorbid psychiatric disorders according to DSM-5 categories. Every interview took about 2 hours to complete. This was a semistructured questionnaire that included the age, area of residence, religion, educational status, employment status, number of family members and family type of the respondents. It also included some questions related to Substance Use like, for how long the adolescent is taking substances, family history and forensic history. The Diagnostic and Statistical Manual of Mental Disorders, Fifth Edition, commonly referred to as the DSM-5, is the latest version of the American Psychiatric Association's gold standard text on the names, symptoms, and diagnostic features of every recognized psychiatric disorder. The DSM 5 criteria for substance use disorders are based on decades of research and clinical knowledge. The DSM 5 recognizes substance use disorders resulting from the use of ten separate classes of drugs: alcohol, caffeine, cannabis, hallucinogens (phencyclidine or similarly acting arylcyclohexylamines), other hallucinogens such as LSD, inhalants, opioids, sedatives- hypnotics- or anxiolytics, stimulants (including amphetamine-type substances, cocaine, and other stimulants), tobacco, and other or unknown substances. Therefore, while some significant grouping of psychoactive substances is accurately identified, use of other or unknown substances can also form the basis of a substance-related or addictive disorder. There are two groups of substance-related disorders: substance use disorders and substance-induced disorders. Substance use disorders are patterns of symptoms resulting from the use of a substance which the individual continues to take, despite experiencing problems as a result. Substance-induced disorders, including intoxication, withdrawal, other substance/medication-induced mental disorders, are detailed alongside substance use disorders. The diagnostic criteria are given in Appendix III. Collected data was processed, 
cleaned and entered into a windows PC. Data were analyzed using statistical software SPSS version 22.0.

\section{Results}

The present study aimed at assessing the presence of comorbid psychiatric disorders among adolescents with Substance Use Disorder (SUD) included a total of 70 adolescents. The tools of assessment were DSM-5 criteria for SUD and Development and Well-Being Assessment (DAWBA) self and parent version. The findings obtained from data analyses are presented below:

Age and other socio-demographic information: Over half
$(60 \%)$ of the respondents were $<15$ years old, and $40 \%$ of the respondents were $>15$ years age. The mean age of the respondents was $13.2 \pm 2.1$ years, and the range was $11-17$ years. More than three-quarters $(81.4 \%)$ of the respondents were Muslim and 14.2\% Hindus. Very few were Christian and Buddhist. Nearly $40 \%$ had a monthly family income of Taka $>30000,27.1 \%$ Taka 20-30 thousand and 21.4\% Taka $10-20$ thousand. Around $66 \%$ of the respondents had in the primary level of education, and $15.7 \%$ had secondary, and $12.8 \%$ were illiterate, and $5.7 \%$ had a non-formal education. Regarding the type of family, $80 \%$ were from a joint family with $20 \%$ having $5-6$ family members (Table 1 ).

Table 1. Distribution of respondents by their socio-demographic condition $(n=70)$.

\begin{tabular}{|c|c|c|}
\hline Socio-demographic characteristics & Frequency $(\%)$ & Mean \pm SD \\
\hline \multirow[t]{2}{*}{ Age (yrs) } & --- & $13.2 \pm 2.1$ \\
\hline & Frequency & Percentage \\
\hline \multicolumn{3}{|l|}{ Religion } \\
\hline Islam & 57 & 81.4 \\
\hline Hindu & 10 & 14.2 \\
\hline Christian & 3 & 4.0 \\
\hline Buddhist & 0 & 00 \\
\hline Monthly income (Tk.) & Frequency & Percentage \\
\hline$<10000$ & 9 & 12.8 \\
\hline $10000-20000$ & 15 & 21.4 \\
\hline $20000-30000$ & 19 & 27.1 \\
\hline$>30000$ & 27 & 38.5 \\
\hline \multicolumn{3}{|l|}{ Education } \\
\hline Illiterate & 9 & 12.8 \\
\hline Primary & 46 & 65.7 \\
\hline Secondary & 11 & 15.7 \\
\hline Non-formal education & 4 & 5.7 \\
\hline \multicolumn{3}{|l|}{ Family type } \\
\hline Nuclear & 56 & 80.0 \\
\hline Joint & 14 & 20.0 \\
\hline \multicolumn{3}{|l|}{ Family members } \\
\hline$<4$ & 24 & 34.2 \\
\hline $5-6$ & 38 & 54.2 \\
\hline 7 or more & 8 & 11.4 \\
\hline
\end{tabular}

Pertinent information about respondents: Over one-quarter $(26.4 \%)$ of the respondents had a family history of mental illness. $6.2 \%$ of respondents had to care for other patients. (Table 2 ).

Table 2. Some pertinent information about respondents $(n=70)$.

\begin{tabular}{lll}
\hline Pertinent information about respondents & Frequency (\%) & Mean \pm SD \\
\hline The family history of mental illness & $21(30.0)$ & --- \\
The family history of substance use & $19(27.1)$ & --- \\
Previous treatment history & $7(10.0)$ & --- \\
Forensic history of any of the parents & $4(1.8)$ & $2.3 \pm 1.4$ \\
Duration of taking illicit substances (yrs) & --- & \\
Any history of trouble with police/conflict with law enforcing agency & $34(48.5)$ & \\
\hline
\end{tabular}

Substance Use Disorders among the respondents: About 50\% of the adolescents interviewed had Tobacco Use Disorder (51.4\%), followed by Cannabis Use Disorder (47.1\%) (Figure 1). 


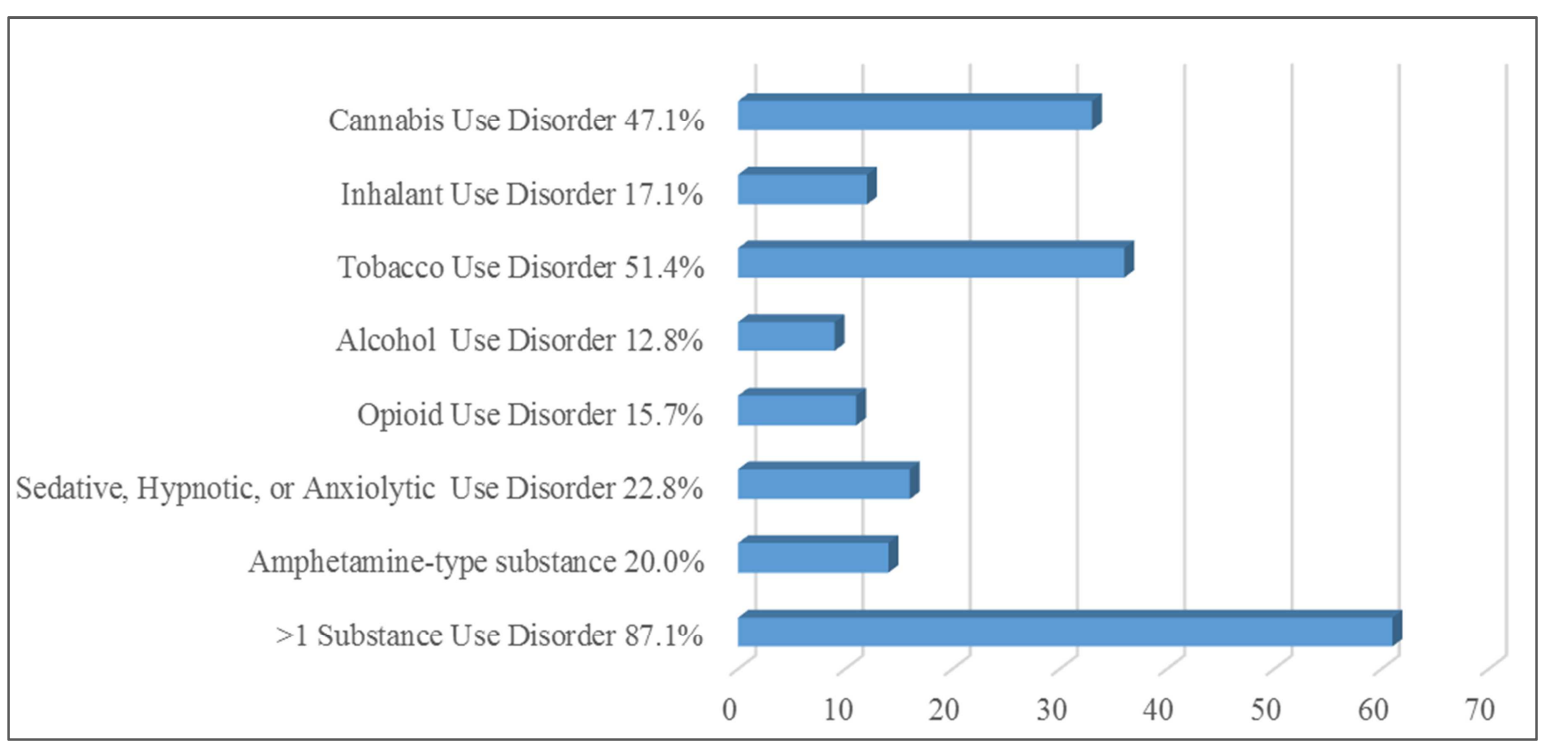

Figure 1. Substance Use Disorders among the respondents $(n=70)$.

Prevalence of psychiatric disorder diagnosed: Among the respondents, $22.8 \%$ had no psychiatric illness. The overall psychiatric disorders among adolescents were $77.1 \%$ (54 out of 70) (Figure 2).

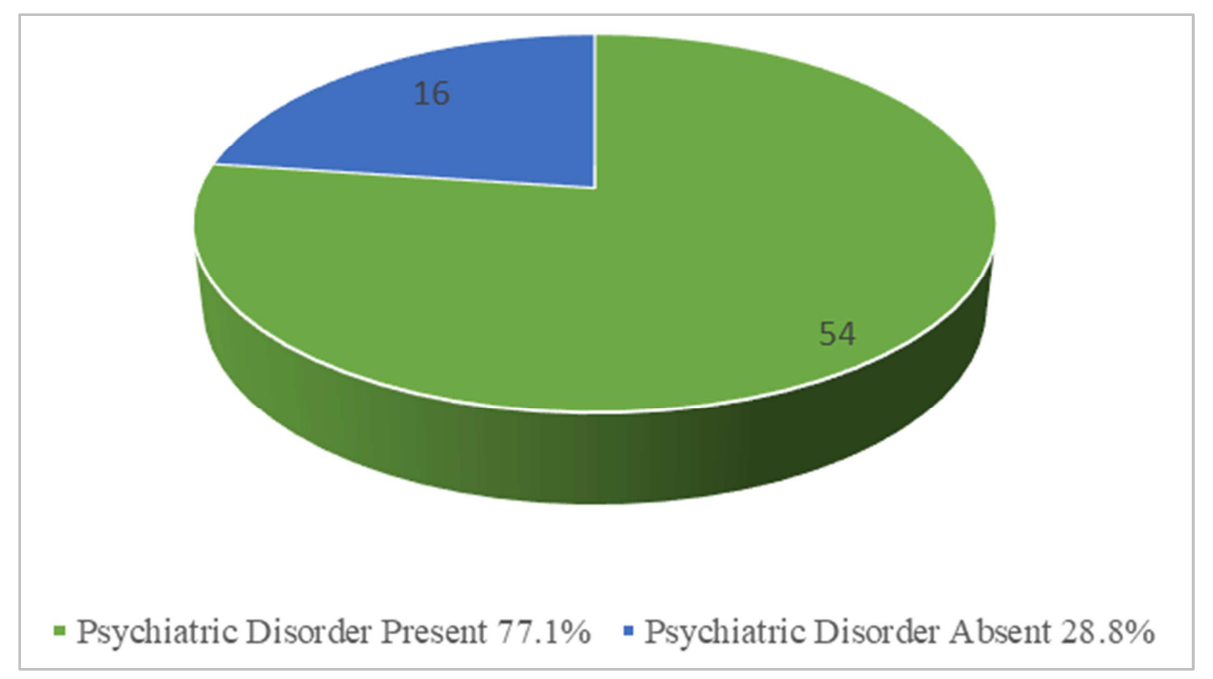

Figure 2. Prevalence of psychiatric disorders among adolescents with SUD $(n=70)$.

Type and frequency of psychiatric disorder diagnosed: Most of the patients (45.7\%) had more than one psychiatric disorder diagnosed. Regarding the specific disorder diagnosed Conduct Disorder was 31.4\%, ADHD in 21.4\%, ODD in 18.5\%, MDD in $17.1 \%$, both Specific and Social Phobia in $10 \%$, GAD in $7.1 \%$, OCD in $5.7 \%$ (Table 3).

Table 3. Pattern of psychiatric disorder diagnosed $(n=54)$.

\begin{tabular}{lll}
\hline Psychiatric disorder & Frequency & Percentage \\
\hline Separation Anxiety Disorder & 2 & 2.8 \\
Specific Phobia & 7 & 10.0 \\
Social Phobia & 7 & 10.0 \\
Panic Disorder & 5 & 7.1 \\
Post-Traumatic Stress Disorder & 2 & 2.8 \\
Obsessive Compulsive Disorder & 4 & 5.7 \\
Generalized Anxiety Disorder & 5 & 7.1 \\
Depressive Disorder & 12 & 17.1 \\
Attention-Deficit/Hyperactivity Disorder & 15 & 21.4 \\
Conduct Disorder & 22 & 31.4 \\
ODD & 13 & 18.5 \\
\hline
\end{tabular}

More than one disorder is considered in one individual. 
Table 4. Association between demographic features and presence of psychiatric disorders $(n=70)$.

\begin{tabular}{|c|c|c|c|}
\hline \multirow{2}{*}{ Demographic features } & \multicolumn{2}{|c|}{ Psychiatric disorder } & \multirow{2}{*}{ p-value } \\
\hline & Present $(n=54)$ & Absent (n=16) & \\
\hline \multicolumn{4}{|l|}{ Age group (yrs.) } \\
\hline $11-13$ yrs. & $22(40.7)$ & $11(68.7)$ & \multirow{2}{*}{0.048} \\
\hline $14-17$ yrs. & $32(59.2)$ & $5(31.2)$ & \\
\hline \multicolumn{4}{|l|}{ Education } \\
\hline Illiterate \& primary & $35(78.5)$ & $09(56.2)$ & \multirow{2}{*}{0.535} \\
\hline Secondary \& other & $19(35.1)$ & $07(43.7)$ & \\
\hline \multicolumn{4}{|l|}{ Income (Taka) } \\
\hline$\leq 300000$ & $33(76.7)$ & $10(62.5)$ & \multirow{2}{*}{0.920} \\
\hline$>30000$ & $21(30.0)$ & $06(37.5)$ & \\
\hline \multicolumn{4}{|l|}{ Religion } \\
\hline Islam & $46(85.1 \%)$ & $11(68.7)$ & \multirow{2}{*}{0.137} \\
\hline Others & $8(14.8)$ & $5(31.2)$ & \\
\hline \multicolumn{4}{|l|}{ Type of family } \\
\hline Nuclear & $42(77.7)$ & $14(87.5)$ & \multirow{3}{*}{0.288} \\
\hline Joint & $14(25.9)$ & $2(12.5)$ & \\
\hline \multicolumn{3}{|l|}{ Family size } & \\
\hline Small (3-4 members) & $18(33.3)$ & $6(37.5)$ & \multirow{2}{*}{0.757} \\
\hline Large (>4 members) & $36(66.6)$ & $10(62.5)$ & \\
\hline
\end{tabular}

*Data were analyzed using $\chi^{2}$ Test. figures in the parentheses denote the corresponding percentage.

Association between demographic characteristics and the presence of psychiatric disorders: Different sociodemographic features were collected like age, education level, family income, religion, family type, and family members and their relationships with the presence of psychiatric disorders was calculated. None showed a significant relationship (Table 4).

Association between other pertinent information and the presence of psychiatric disorders: Different pertinent information about the respondents was collected and was analyzed to see any relationships between the presence and absence of psychiatric disorders. The family history of mental illness and Family history of substance use shows no significant relationships. Significant differences were found between the adolescents with and without any history or trouble with law enforcing agency (Table 5).

Table 5. Association between other pertinent information and presence of psychiatric disorders $(n=70)$.

\begin{tabular}{lll}
\hline Pertinent information about respondents & \multicolumn{2}{l}{ Psychiatric disorder } \\
\cline { 2 - 3 } & Present (n=54) & Absent (n=16) \\
\hline The family history of mental illness & & \\
Present & $15(27.7)$ & $10(62.5)$ \\
Absent & $39(72.2)$ & \\
The family history of substance use & & $4(25.0)$ \\
Present & $15(27.7)$ & $10(62.5)$ \\
Absent & $39(72.2)$ & \\
Any history of trouble with police /conflict with law enforcing agency & & $6(11.1)$ \\
Present & $28(22.8)$ & 0.456 \\
Absent & $19(24.1)$ & $17(11.8)$ \\
\hline
\end{tabular}

*Data were analyzed using $\chi^{2}$ Test. figures in the parentheses denote the corresponding percentage.

\section{Discussion}

Adolescence is often described as a time of experimentation with "risky" or "problem" behaviors [20], and substance use is one such behavior that is initiated during this age period. Substance use and addictive disorders are topics of considerable importance both because of their significance for adolescent development and because of their public health impact. The impact and management of adolescent SUD are further complicated by other comorbid psychiatric disorders. Many studies have systematically documented that there are adolescents with SUD suffers from more burden of other psychiatric disorder than that of the general adolescents. The present study aimed to determine the pattern of comorbid psychiatric disorders among the admitted adolescents of two institutions namely Central Drug Addiction Treatment Center (CDC), Tejgaon, Dhaka, and the Ashokti Punorbashon Nibash (APON) in Singair, Manikganj, Bangladesh. The authority of the two institutions was helpful regarding the data collection. The researcher wanted to collect multisource data namely from parents and the adolescents to confirm the diagnosis of comorbid psychiatric disorders. But both the centers keep the adolescents alone take necessary cares by themselves. So at first, it was difficult to collect the parent data. This problem was solved 
at $\mathrm{CDC}$ by interviewing the parents just after admission and when they come to see their child. Moreover, in APON there is specific parent meeting date and parental counseling date twice monthly. So the parents were approached by the researcher at that specific date. Thus the multisource data collection was ensured. Over half $(60 \%)$ of the respondents were $<15$ years old, and $40 \%$ of the respondents were $>15$ years age. The mean age of the respondents was 13.2 \pm 2.1 years with a range of 11-17 years. Only male patients were taken as the sample because the above-mentioned treatment facilities do not provide service for the female adolescents. More than three-quarters $(81.4 \%)$ of the respondents were Muslim, and nearly $60 \%$ had a monthly family income of less than taka 30000 , Around $66 \%$ of the respondents had in the primary level of education, and $15.7 \%$ had secondary, and $12.8 \%$ were illiterate, and $5.7 \%$ had non-formal education. Regarding the type of family, $80 \%$ were from a joint family with $20 \%$ having $5-6$ family members. Regarding the pattern of SUD among the adolescents most common was the Tobacco Use Disorder $(51.4 \%)$, followed by Cannabis Use Disorder (47.1\%), Sedative, Hypnotic, or Anxiolytic Use Disorder (22.8\%), Amphetamine-type substance $(20.0 \%)$, Inhalant Use Disorder (17.1\%), Opioid Use Disorder (15.7\%), Alcohol Use Disorder (12.8\%), >1 Substance Use Disorder (87.1\%). There were no cases of Hallucinogen Use Disorder. Data from the Monitoring the Future (MTF) study in the US, a national school-based survey, show that adolescent substance use is relatively common by the end of the high school years. Cannabis was the most frequently used illegal drug, with $31.5 \%$ of 12 th graders reporting some lifetime use [21]. Alcohol and tobacco are even more common, with $73 \%$ of today's students have consumed alcohol by the end of high school and $45 \%$ of high school seniors reporting drinking in the past month [22]. Forty-seven percent of high school seniors report some experience with cigarette smoking, and $22 \%$ were current smokers in 2006 [23]. The use of different drugs is highly interrelated in both epidemiological and clinical samples of adolescents [20]. For example, in the 1985 National Household Survey on Drug Abuse (NHSDA) data, $24 \%$ of illicit drug users used multiple drugs simultaneously within the past year, and $43 \%$ had used alcohol along with an illicit drug [22]. Found that males, older youth, and the U.S. born participants were more likely to report using multiple substances than females, younger youth, and foreign-born participants [23]. Another community based study done among the adolescents in the US in 2007 using DSM-IV shows that Any substance use disorder 5.3\% where Alcohol abuse or dependence was $2.9 \%$, Marijuana abuse or dependence $3.4 \%$, other substances abuse or dependence $0.9 \%$, any substance abuse $3.9 \%$, any substance dependence $2.2 \%$, one or more diagnoses $17.1 \%$ [24]. A study was done among the street children in Mumbai, India shows the prevalence of Substance Use Disorder is around 90\%. Nicotine was the most frequent substance of abuse, used by 104 (63.8\%) adolescent street children. Seventy-eight (48\%) adolescents were using inhalants. Sixty (37\%) were using alcohol, and $42(26 \%)$ were using sedatives and stimulants. Thirty-one (19\%) were found to be using cannabis and opioids [25]. Review literature from other Indian study shows a pattern of SUD. Data from student and non-student populations indicate that alcohol, tobacco, minor tranquilizer, analgesics and cannabis are the standard drugs being abused [22]. Among the 70 adolescents interviewed 54 (77.1\%) was diagnosed with having a comorbid psychiatric disorder. The rate of psychiatric disorder is much higher than that of the general adolescents. Early epidemiological studies indicated that, during the course of one year, $5 \%$ to $15 \%$ of children between 9 and ten years of age presented behavioral or emotional disorders with functional impairment [26]. Illustrating the difficulty of obtaining accurate epidemiological rates in child psychiatry, a recent review including studies from the last four decades, showed a considerable variation in prevalence estimates ( $1 \%$ to $51 \%)$. It also illustrated the diversity in diagnostic criteria adopted, which initially were the Rutter criteria and label the ICD and DSM criteria [27]. According to the analytical predictions, prevalence would be roughly $10-20 \%$ among children and adolescents as like as the prevalence findings of the reports of the developing countries. A study of psychiatric outpatient attendance at the Institute of Mental Health and Research in Dhaka, revealed that $8.6 \%$ of cases were children or adolescents [10]. In a different analysis of psychiatric morbidity among the Institute outpatients, emotional disorder was found to be the largest group with $32.5 \%$, followed by conduct disorder $18.8 \%$; mental retardation comprised $16.2 \%$, psychoses and allied conditions $11.2 \%$, epilepsy with behavioral problems $12.5 \%$ and the rest $8.5 \%$ comprised of other groups of disorders, according to ICD-9 criteria [11]. A multicentric exploratory study to assess the prevalence of psychiatric disorders among 5-10-year-olds in rural, urban and slum areas in Bangladesh by Mullick and Goodman found the overall prevalence is $15.2 \%$ [11]. Rabbani et al., [12] shows the prevalence of mental disorders among children adolescents aged 5-17 years is $18.4 \%$. So it is evident that the burden of the comorbid psychiatric disorder is found very high among adolescents with SUD. Most common disorder found was Conduct Disorder (CD) 31.4\%, Attention-Deficit/Hyperactivity Disorder (ADHD) 21.4\%, Oppositional Defiant Disorder (ODD) 18.5\%, Major Depressive Disorder (MDD) 17.1\%, Social Phobia 10\%, Specific Phobia 10\%, Panic Disorder 7.1\%, Generalized Anxiety Disorder (GAD) 7.1\%, Obsessive Compulsive Disorder (OCD) 5.7\%, Post Traumatic Stress Disorder (PTSD), and Separation Anxiety Disorder 2.8\%. Methods for the Epidemiology of Child and Adolescent Mental Disorders (MECA) Study found $29.8 \%$ for anxiety disorders, $7.9 \%$ for mood disorders and $18.7 \%$ for disruptive behaviors. The study also showed that among adolescents with current SUD, $76.0 \%$ (70.0\% of females, $80.0 \%$ of males) also had anxiety, mood, or disruptive behavior disorder compared with $24.5 \%$ of adolescents without current SUD, [28] described several possible relationships between adolescent substance abuse and affective disorders, conduct disorder and antisocial 
personality, anxiety disorders, attention-deficit hyperactivity disorder, schizophrenia and psychotic symptoms, and eating disorders. Among alcohol-abusing and alcohol-dependent patients, prevalence rates for psychiatric comorbidity of between $57 \%$ and $84 \%$ have been reported which is quite similar to the present study [16]. There is a growing body of research on COD among adolescents. However, most information has emerged from studies conducted in the United States. A US survey investigated the prevalence of SUD among 203 adolescent psychiatric inpatients and found $41 \%$ met the criteria for a SUD [4]. Another investigated adolescent admissions to residential substance abuse program and reported the prevalence of psychiatric disorders comorbidity in $64 \%$ of the 91 adolescents; depression $(24 \%)$, conduct disorder (CD) (24\%) and attention deficit hyperactivity disorder (ADHD-11\%) were the most common conditions [14]. Further, literature review estimate rates of psychiatric comorbidity among adolescents receiving treatments for substance abuse at 50-90\% [15]. The high levels of psychiatric comorbidity among adolescents with SUD suggest dual diagnosis is 'the rule rather than the exception' [16]. Adolescents with SUD have higher rates of both mood disorders and $\mathrm{CD}$ [15], attention deficit hyperactivity disorder, $\mathrm{CD}$ and substance use often co-exist and are associated with poorer treatment outcomes, [14] those with $\mathrm{CD}$ are more likely to have an earlier onset of substance use [16], timely intervention of co-occurring psychiatric and SUDs is associated with better engagement with treatment [17]. The study has some limitations. Female participants could not be included, and the study included only two centers where usually problematic children and adolescents are admitted. So the study result cannot be generalized. Another limitation is in the methodology where SUD is diagnosed with DSM-5 criteria, where a structured interview could give a more accurate diagnosis.

\section{Conclusion \& Recommendations}

Based on the findings of the study, it can be concluded that adolescents with SUD have a high rate of other comorbid psychiatric disorders. Understanding the relationship in etiological perspective and variables which influences the problem will help to provide intervention services for adolescents affected by SUD. The adolescent is high time for developing SUD and if the proper intervention can be started at this stage. Therefore, it can be recommended that during the management of Substance Use Disorder among the adolescents more emphasis should be given in the comorbid psychiatric disorders. Further epidemiological studies are needed to get a more representative result.

\section{References}

[1] Thapar, A., Taylor, E., Snowling, M., Scott, S., Leckman, J. and Pine, D. (2015). Rutter's child and adolescent psychiatry. Malden, MA: Wiley-Blackwell.
[2] Tripathi, B. and Lal, R. (1999). Substance abuse in children and adolescents. Indian J Pediatr, 66 (4), pp. 569-575.

[3] Older, J. (1986) .Anti-smoking language that the young understand. World health forum, 7 (1): pp 74-78.

[4] Piazza, N. (1996). Dual Diagnosis and Adolescent Psychiatric Inpatients. Substance Use \& Misuse, 31 (2), pp. 215-223.

[5] Pal, R., Dasgupta, A. and Tsering, D. (2010). Substance use among adolescent high school students in India: A survey of knowledge, attitude, and opinion. Journal of Pharmacy and Bioallied Sciences, 2 (2), pp. 137.

[6] Griswold, K. S., Aronoff, H., Kernan, J. B., Kahn, L. S. (2008) Adolescent substance use and abuse: recognition and management. Am Fam Physician. 2008; 77 (3): pp. 331-336.

[7] Shantna, K., Chaudhury, S., Verma, A. and Singh, A. (2009). Comorbid psychiatric disorders in substance dependence patients: A control study. Industrial Psychiatry Journal, 18 (2), p. 84 .

[8] O'Neil, K., Conner, B. and Kendall, P. (2011). Internalizing disorders and substance use disorders in youth: Comorbidity, risk, temporal order, and implications for intervention. Clinical Psychology Review, 31 (1), pp. 104-112.

[9] Wu, L., Gersing, K., Burchett, B., Woody, G. and Blazer, D. (2011). Substance use disorders and comorbid Axis I and II psychiatric disorders among young psychiatric patients: Findings from a large electronic health records database. Journal of Psychiatric Research, 45 (11), pp. 1453-1462.

[10] Mullick, M. S. I., Khanam, M., Islam, H. (1995). Psychiatric morbidity of outpatient children in Institute of Mental Health and Research. Bang J Psychiatry, 7, pp. 4-8.

[11] Rabbani M. G., Alam M. F., Ahmed HU, Sarker M, Chowdhury WA, Zaman MM et al. (2009). Prevalence of mental disorders, mental retardation, epilepsy and substance abuse in children. Bang J Psychiatr, 23, pp 13-54.

[12] Davis, L., Uezato, A., Newell, J. and Frazier, E. (2008). Major depression and comorbid substance use disorders. Current Opinion in Psychiatry, 21 (1), pp. 14-18.

[13] Wise, B., Cuffe, S. and Fischer, T. (2001). Dual diagnosis and successful participation of adolescents in substance abuse treatment. Journal of Substance Abuse Treatment, 21 (3), pp. 161-165.

[14] Solhkhah, R. (2003). The intoxicated child. Child and Adolescent Psychiatric Clinics of North America, 12 (4), pp. 693-722.

[15] Dias, D. and Brown, E. (2006). Adolescent Substance Abuse and Psychiatric Comorbidities. The Journal of Clinical Psychiatry, 67 (07), p. e02.

[16] Chi, F., Sterling, S. and Weisner, C. (2006). Adolescents with Co-Occurring Substance Use and Mental Conditions in a Private Managed Care Health Plan: Prevalence, Patient Characteristics, and Treatment Initiation and Engagement. American Journal on Addictions, 15 (s1), pp. 67-79.

[17] Chisolm, D., Mulatu, M. and Brown, J. (2009). Racial/ethnic disparities in the patterns of co-occurring mental health problems in adolescents in substance abuse treatment. Journal of Substance Abuse Treatment, 37 (2), pp. 203-210. 
[18] Edokpolo, O., James, P., Kearns, C., Campbell, A. and Smyth, B. (2010). Gender Differences in Psychiatric Symptomatology in Adolescents Attending a Community Drug and Alcohol Treatment Program. Journal of Psychoactive Drugs, 42 (1), pp. 31-36.

[19] Steinberg, L. (2007). Risk Taking in Adolescence. Current Directions in Psychological Science, 16 (2), pp. 55-59.

[20] Johnston, L., Malley, P., Bachman, J. (2000). Monitoring the Future: National survey results on drug use, 1975- 1999. (NIH Publication No. 00- 4802). Bethesda, MD: National Institute on Drug Abuse.

[21] Johnston, L. D., Malley, P. M., Bachman, J. G., \& Schulenberg, J. E. (2007). Monitoring the Future national results on adolescent drug use: Overview of key findings, 2006 (NIH Publication No. 07 - 6202). Bethesda, MD: National Institute on Drug Abuse.

[22] Mohan D, et al. (1992) Integrating alcohol, tobacco and other drugs in survey research. Department of Psychiatry and Drug Dependence Treatment Centre, New Delhi.

[23] Sneed, C., Morisky, D., Rotheram-Borus, M., Lee, S. and Ebin, V. (2004). Indices of lifetime polydrug use among adolescents. Journal of Adolescence, 27 (3), pp. 239-249.
[24] Roberts, R., Roberts, C., Xing, Y. (2007). Comorbidity of substance use disorders and other psychiatric disorders among adolescents: Evidence from an epidemiologic survey. Drug and Alcohol Dependence, 88, pp. S4-S13.

[25] Roberts, R. E., Attkisson, C. C., Rosenblatt, A. (1998). Prevalence of psychopathology among children and adolescents. American Journal of Psychiatry, 155, pp 715-725.

[26] Gaidhane, A., Syed Zahiruddin, Q., Waghmare, L., Shanbhag, S., Zodpey, S. and Joharapurkar, S. (2008). Substance abuse among street children in Mumbai. Vulnerable Children and Youth Studies, 3 (1), pp. 42-51.

[27] Simonoff E., Pickles A., Meyer J. J., Silberg J. L., Maes H. H., Loe ber R., Rutter M., Hewitt J. K. \& Eaves, L. J. (1997). The Virginia Twin Study of Adolescent Behavioral Development: influences of age, gender and impairment in rates of disorder. Archives of General Psychiatry, 54, pp. 801-808.

[28] Kandel, D., Johnson, J., Bird, H., Weissman, M., Goodman, S., Lahey, B., Regier, D. And Schwab-Stone, M. (1999). Psychiatric comorbidity among adolescents with substance use disorders: findings from the MECA study. Journal of the American Academy of Child \& Adolescent Psychiatry, 38 (6), pp. 693-699. 